\title{
Microscopic engine powered by critical demixing
}

\author{
Falko Schmidt ${ }^{a}$, Alessandro Magazzù $^{b}$, Agnese Callegari $^{b}$, Luca Biancofiore $^{c}$, Frank \\ Cichos $^{d}$, and Giovanni Volpe ${ }^{a, b}$ \\ ${ }^{a}$ Department of Physics, Gothenburg University, Origovgen 6 B, 41296 Göteborg, Sweden, \\ ${ }^{b}$ Department of Physics, Bilkent University, 06800 Ankara, Turkey, \\ ${ }^{c}$ Department of Mechanical Engineering, Bilkent University, 06800 Ankara, Turkey, \\ ${ }^{d}$ Department of Physics and Geosciences, University of Leipzig, Linnéstrasse 5, 04103 Leipzig, Germany
}

falkoschmidt@icloud.com

\begin{abstract}
We propose a new type of engine that is powered by the local, reversible demixing of a critical binary liquid. A microscopic particle is optically trapped and performs revolutions due to the emergence of diffusiophoresis.
\end{abstract}

OCIS codes: $350.4855,350.4990$.

During the last few decades much effort has gone into the miniaturization of machines down to the microscopic scale with robotic solutions indispensable in modern industrial processes and play a central role in many biological systems. There has been a quest in understanding the mechanism behind molecular motors and several approaches have been proposed to realize artificial engines capable of converting energy into mechanical work. These current micronsized engines depend on the transfer of angular momentum of light, are driven by external magnetic fields, due to chemical reactions or by the energy flow between two thermal reservoirs [1-4]. Here we propose a new type of engine that is powered by the local, reversible demixing of a critical binary liquid. In particular, we show that an absorbing, optically trapped particle performs revolutions around the optical beam because of the emergence of diffusiophoresis and thereby produces work. This engines is adjustable by the optical power supplied, the temperature of the environment and the criticality of the system.

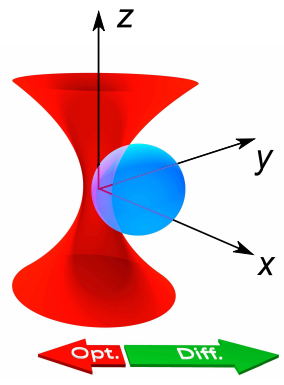

(a)

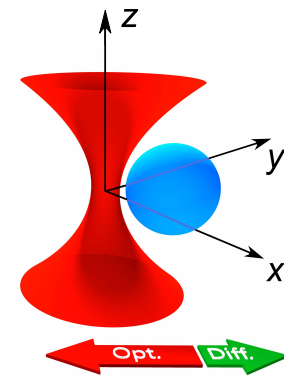

(b)

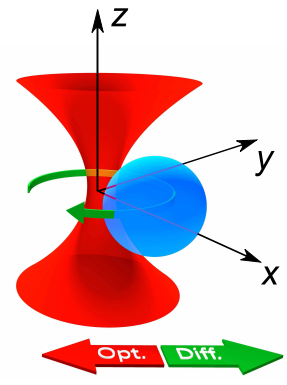

(c)

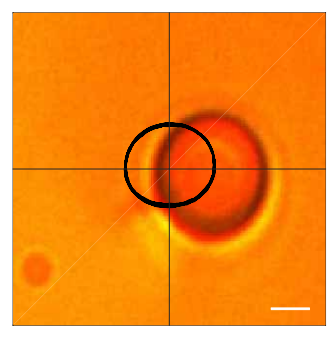

(d)

Fig. 1. (a-c) An optically trapped microsphere experiences a harmonic restoring optical force that attracts it towards the center of the optical trap near the focal spot (red arrows). If the particle absorbs the illumination light, the temperature of the surrounding fluid increases generating an asymmetric temperature profile which is hotter on the side of the particles closer to the focal spot. The temperature gradient, in turn, generates a concentration gradient surrounding the particle and, eventually, a diffusiophoretic force (green arrows). (d) Microscopic image of an absorbing particle with circular trajectory in $x-y$ plane. Scale bar: $1 \mu \mathrm{m}$.. 


\section{References}

1. P. A. Quinto-Su, "A microscopic steam engine implemented in an optical tweezers", Nat. Comm. 5, (2014).

2. V. Blickle, C. Bechinger, "Realization of a micrometre-sized stochastic heat engine," Nat. Phys. 8, 143-146 (2012).

3. I. A. Martinez Blickle, E. Roldan, L. Dinis, D. Petrov, J. M. R. Parrondo, R. A. Rica, "Brownian Carnot engine," Nat. Phys. 12, 67-70 (2016).

4. S. L. Neale, M. P. MacDonald, K. Dholakia, and T. F. Krauss, "All- optical control of microfluidic components using form birefringence," Nat. Mat. 4, 530-533 (2005). 